\section{Identità di genere in pediatria}

\author{
Intervista di Chiara Centenari ${ }^{1}$ \\ a Gianluca Tornese ${ }^{2}$
}

Pediatra, Ospedale Versilia; membro del Gruppo di Studio

"Pediatria di genere" dell'ACP

2 Pediatra Endocrinologo, Ambulatorio Pediatrico per la Varianza di Genere (APEVAGE), Istituto per l'infanzia IRCCS "Burlo Garofolo" di Trieste

\section{Cosa è il servizio multidisciplinare APEVAGE di Trieste e quali pazienti vengono presi in carico?}

L'Ambulatorio Pediatrico per la Varianza di Genere è uno dei pochi servizi multidisciplinari presenti in Italia che si occupa della presa in carico di minori con varianza di genere o con sospetta diagnosi di disforia di genere. I minori con "varianza o non conformità di genere" sono bambini/e che presentano espressioni e ruoli di genere (abbigliamento, giochi, attività, sport) che non si allineano allo standard binario maschile/ femminile atteso in base al sesso di appartenenza. Si parla invece di "disforia di genere" quando il minore non si identifica nel genere attribuito alla nascita o sente addirittura di appartenere al genere opposto esprimendo disforia (dal greco, "sopportare male") anatomica e vivendo questa incongruenza come una sofferenza profonda che si ripercuote sulla sua qualità di vita [Tabella 1]. Il nostro team è nato nel 2015 ed è formato da un pediatra endocrinologo, due psicologhe, una neuropsichiatra infantile e una bioeticista. I pazienti giungono al servizio su richiesta del medico curante, di uno psicologo/psichiatra/NPI o per accesso diretto del minore con la famiglia. La prima visita avviene sempre con il pediatra e rappresenta un incontro di conoscenza per analizzare la situazione, far emergere le richieste del minore e/o della famiglia e spiegare in cosa consiste la presa in carico. Il fatto di avere come primo contatto il pediatra è generalmente molto rassicurante per la famiglia, rimuovendo quello stigma purtroppo ancora esistente legato alle visite psicologiche/psichiatriche che può ritardare la gestione del paziente. La successiva presa in carico, previo consenso di entrambi i genitori, è strutturata in una visita medica e una valutazione psicologica/NPI. Per la parte medica, se ritenuti necessari, vengono prescritti approfondimenti endocrinologici/genetici per escludere eventuali patologie concomitanti quali i Disturbi della Differenziazione Sessuale (DSD) che frequentemente si associano a disforia fin dalla prima infanzia. La valutazione NPI e psicologica con l'ausilio di questionari serve per definire la sola presenza di una varianza di genere o di una vera a propria disforia e per escludere comorbidità psichiatriche. I criteri diagnostici della disforia di genere per bambini e adolescenti sono classificati dal DSM 5 [1]. In base alla diagnosi e all'età del paziente viene deciso con la famiglia il percorso che consiste nella presa in carico psicologica e, in casi selezionati, nella terapia ormonale indirizzata a ritardare lo sviluppo puberale (bloccanti ipotalamici analoghi LHRH - triptorelina) e/o a finalizzare il cambiamento di sesso (testosterone/estrogeni) secondo protocolli condivisi.

\section{In Italia quali altri presidi simili ci sono? Esiste una rete con protocolli condivisi?}

In Italia sono pochi i centri come il nostro che si occupano di minori, questi si trovano a Bari, Bologna, Napoli, Roma, To- rino e Firenze e afferiscono alla rete nazionale ONIG (Osservatorio Nazionale Identità di Genere) [2] costituita da team di esperti che condividono percorsi diagnostico-terapeutici per adulti e minori rifacendosi agli standard di cura del World Professional Association of Transender Health (WPATH) [3]. All'interno dell'ONIG esiste un gruppo di studio che si occupa di bambini e adolescenti; le linee-guida italiane pediatriche attualmente sono in via di revisione. Per quanto riguarda i minori i trattamenti ormonali, pur essendo previsti da linee guida nazionali e internazionali, sono oggetto di dibattito medico e bioetico. Tuttavia è necessario tener presente che ritardare il trattamento fino alla tarda adolescenza o all'età adulta potrebbe aggravare il quadro psicologico del minore. L'intervento con bloccanti ipotalamici è reversibile e può essere considerato come un tempo di "vigile attesa e di diagnosi estesa" durante il quale il minore valuta meglio la propria situazione riducendo lo stress dato dal cambiamento del corpo e il relativo rischio di incorrere in complicanze psichiatriche. Se viene poi confermata la volontà di iniziare il percorso di riassegnazione di genere, raggiunti i I 6 anni è possibile l'induzione della pubertà con estrogeni o testosterone per sviluppare i caratteri sessuali secondari del genere percepito come proprio dall'adolescente.

Tabella 1. Definizioni di varianza e disforia di genere

Varianza di genere Quando l'identità di genere o il ruolo di genere di un individuo si differenziano dalle norme culturali comuni per una persona di un determinato sesso.

Disforia di genere Quando l'incongruenza tra la propria identità di genere e il genere assegnato alla nascita (e il ruolo associato a quel genere e/o a quelle caratteristiche sessuali primarie o secondarie) si associa a sofferenza clinicamente significativa 0 a compromissione del funzionamento in ambito sociale, scolastico o in altre aree importanti.

Quali sono i criteri di invio da parte dei pediatri di libera scelta? E quale formazione è necessaria ai colleghi per individuare bisogni di minori e famiglie?

È esperienza comune che i pediatri non ricevono alcuna formazione specifica sulle "questioni di genere" durante il loro percorso formativo universitario e professionale. Nella maggior parte dei casi quindi si possono sentire impreparati ad affrontare tali situazioni e la loro capacità di intervento è basata principalmente sull'esperienza/conoscenza personale dell'argomento e sulle loro doti di empatia/capacità comunicativa con ragazzi e famiglia.

I pediatri di libera scelta hanno però un ruolo fondamentale nel far emergere, intercettare e accogliere sofferenze del minore o dubbi della famiglia rispetto a questioni di genere. Se la famiglia esprime una preoccupazione circa un "comportamento non conforme" del/la figlio/a sarebbe auspicabile non minimizzare ma esplorare più in dettaglio la situazione per poi dare risposte competenti o, se valutato necessario, indirizzare a specialisti. In particolare sarebbe bene che i pediatri ponessero particolare attenzione ad alcuni "campanelli di allarme":

- bambini il cui comportamento si discosta da quello atteso in base al sesso (varianza di genere) che potrebbero essere vittime di pregiudizi o i cui genitori manifestano preoccupazione a riguardo richiedono supporto;

- disturbi somatoformi (cefalea, dolore addominale, astenia) non riconducibili a patologia organica ma da considerare 
Tabella 2. Vocabolario per le "questioni di genere"

$\begin{array}{ll}\text { Sesso biologico } & \begin{array}{l}\text { Appartenenza al sesso maschile o femminile } \\ \text { data dai cromosomi sessuali e dagli ormoni che } \\ \text { determinano il sesso gonadico e il fenotipo }\end{array}\end{array}$

Identità di genere Percezione che ognuno ha del proprio sentirsi maschio o femmina

Ruolo di genere

Orientamento sessuale
"A quale sesso corrisponde la biologia del mio corpo?"

\begin{abstract}
"A quale categoria sento di appartenere intimamente e psichicamente?"
\end{abstract}

\begin{abstract}
"Che cosa penso di dover fare/cosa voglio fare, e che cosa ci si aspetta da me, in quanto maschio o in quanto femmina?"
\end{abstract}
"A quale sesso e/o genere appartengono le persone che mi attraggono emotivamente, affettivamente e fisicamente?"

spia di sofferenza psicologica eventualmente legata a "questioni di genere";

- Disturbi del Comportamento Alimentare (DCA) soprattutto nei maschi, visto che l'ipogonadismo ipogonadotropo da carenza nutrizionale causa un'inibizione "naturale" della pubertà (e tale informazione è facilmente reperita dai ragazzi tramite internet);

- minori che esprimono disagio con il proprio corpo da maschio/femmina (disforia);

- automutilazioni o condotte a rischio (abuso di droghe, alcol).

Sarebbe auspicabile che in ogni Regione fosse concordato un percorso diagnostico terapeutico condiviso tra pediatri di libera scelta e le strutture specialistiche di riferimento per le "questioni di genere".

\section{Ci sono strumenti utili e fruibili per $i$ pediatri? Quale $\dot{e}$ l'opportuno vocabolario da utilizzare?}

Sicuramente è importante avere conoscenza del corretto vocabolario e delle relative definizioni con cui riferirsi alle "questioni di genere" [4]. In particolare avere ben chiari i concetti di "sesso biologico", "identità di genere", "ruolo di genere" e "orientamento sessuale" [Tabella 2]. È necessario illustrare questi concetti agli studenti di medicina e ai congressi pediatrici anche con semplici esempi che possono riguardare usanze culturali. Per esempio in Africa per due amiche non è possibile camminare tenendosi per mano mentre è consuetudine tra uomini eterosessuali come segno di amicizia. In Occidente notoriamente questo comportamento tra coppie di uomini è invece considerato espressione di omosessualità. Così come l'uso di gonne/pantaloni/orecchi$\mathrm{ni} /$ trucco/colore rosa-blu è variabile nel tempo e nelle culture ed è legato all'espressione sociale del genere non necessariamente coincidente con sesso/identità e orientamento. Anche in Italia questi costumi sono cambiati molto negli ultimi decenni nella direzione di una società meno, ma pur sempre, legata a stereotipi maschile/femminile nell'abbigliamento e nei ruoli di genere.

La modalità e la tempistica con cui il pediatra può indagare queste sfere della personalità di un/a bambino/a o adolescente sono difficilmente riassumibili in formule o domande standardizzate. Rimane quindi delegata all'esperienza e alle capacità del medico stesso il quale dovrebbe porsi sempre in posizione neutrale, evitando di dare per scontata l'identità di genere e l'orientamento sessuale dei minori con le relative aspettative sociali. È importante durante le visite evitare di connotare con rigidi stereotipi l'appartenenza al genere maschile o femminile; per esempio non dare per scontato che tutte le bambine siano "principesse" e amino il rosa, oppure che i bambini non pratichino danza, oppure che chiedere a un adolescente della fidanzata sia sempre per lui motivo di orgoglio.
Quali sono i rischi di salute psico-fisici per questi pazienti soprattutto se viene ritardata la presa in carico?

Solamente una minoranza dei bambini con varianza di genere presenta poi nella vita una vera e propria disforia pertanto le eventuali sofferenze che possono sperimentare sono essenzialmente legate a come il mondo esterno fa loro vivere questa diversità. È essenziale quindi la de-patologizzazione di espressioni di genere non conformi e la massima accoglienza dei minori in tutte le loro modalità espressive anche se non seguono i rigidi stereotipi maschile/femminile culturalmente attesi. Il pediatra dovrebbe porre particolare attenzione a questi bambini perché proprio per il loro "apparire diversi" sono a maggior rischio di fenomeni quali maltrattamento psico-fisico, abuso sessuale e patologia ansioso-depressiva [5]. Per quanto riguarda minori con vera e propria disforia di genere gli studi dimostrano che a causa della loro sofferenza presentano una ridotta qualità di vita e sperimentano secondarie patologie ansioso-depressive, maggiore incidenza di errati stili di vita (abuso di alcol, droghe, partner multipli) e tentativo di suicidio. Recenti studi dimostrano come pazienti a cui non viene fornita la terapia di inibizione della pubertà presentano una prevalenza di ideazione e tentativo di suicidio significativamente più elevata del gruppo trattato [6]. Quindi "il non fare" non è una scelta neutra bensì è causa di complicanze negative sulla vita di questi ragazzi.

\section{Quanto è importante sensibilizzare famiglie e società ver- so l'abbattimento di rigidi stereotipi di genere educativi e l'accoglienza del minore in tutte le sue "diversità"?}

Questa è una parte fondamentale perché se la nostra società fosse più aperta $\mathrm{d}$ accogliente molti dei problemi di questi ragazzi sparirebbero. Per quanto riguarda il nostro centro possiamo dire che la casistica è prevalentemente costituita da adolescenti con sesso assegnato alla nascita femminile che desiderano intraprendere un percorso di transizione verso il sesso opposto cioè FTM (Female to Male). Tale maggiore prevalenza di FTM rispetto a MTF (Male to Female) è confermata anche da casistiche internazionali. Sappiamo bene che quello che noi vediamo è solo la punta di un iceberg sommerso a causa dello stigma sociale che spesso non permette ai minori di emergere con le loro richieste. I genitori non sono quasi mai allineati con i desideri dei figli, ma anche se non capiscono il loro desiderio comprendono che per i ragazzi è una sofferenza e si affidano ai professionisti sanitari. Una delle prime cose che facciamo è spiegare alla famiglia e ai ragazzi la terminologia e i significati di sesso, identità di genere, ruolo di genere $\mathrm{e}$ orientamento sessuale così che possano almeno conoscere le coordinate di base per orientarsi correttamente. Avere quindi la famiglia vicina che accompagna i minori nei loro percorsi è fondamentale per ridurre l'incidenza di complicanze psicologiche/psichiatriche, condotte nocive e rischio di suicidio dei 
minori stessi. Per quanto riguarda il mondo esterno che i minori si trovano ad affrontare sarebbe importante che la società e la scuola fossero accoglienti senza farli sentire sbagliati perché non conformi a rigidi stereotipi di genere.

Educare gli studenti e formare il personale scolastico è quindi fondamentale per raggiungere questo obiettivo. Per ridurre fonti di stress per gli studenti con disforia di genere e/o in transizione sarebbe per esempio auspicabile che il servizio scolastico accogliesse le richieste dei minori per quanto concerne il nome con cui si vogliono far chiamare (es. Luca invece di Lucia) oppure essere chiamati usando il cognome, così come far attenzione all'uso di pronomi maschile/ femminile. Nella nostra esperienza i dirigenti scolastici, pur in assenza di una normativa specifica, generalmente accolgono le richieste di carriera alias che può essere richiesta dalla famiglia di un alunno minorenne che dimostri di aver intrapreso un percorso psicologico e/o medico teso a consentire l'eventuale rettificazione di attribuzione di sesso e desideri utilizzare un nome diverso da quello anagrafico, definito appunto alias.

\section{Confrontandoci con il resto d'Europa come si colloca la percezione delle "questioni di genere dei minori" in Italia?} In Italia riscontriamo ancora molta reticenza culturale ad affrontare questi temi in ambito sociale, educativo e socio-sanitario mentre per quanto riguarda le terapie ormonali per la disforia di genere nei minori siamo all'avanguardia. Questo è stato possibile grazie a due determine emanate dall'Agenzia Italiana del Farmaco (AIFA) nel 2019 e nel 2020 che regolano prescrittibilità e rimborsabilità dei bloccanti ipotalamici $[7] \mathrm{e}$ della terapia cross sex [8], in precedenza considerati per i minori off-label e a pagamento. In aggiunta per quanto riguarda la prescrizione della triptorelina la determina AIFA del 20I9 rende obbligatoria la presenza della figura del bioeticista nell'équipe per coadiuvare medici e psicologi nella valutazione del caso. In altri Paesi, invece, sono più indietro o si stanno facendo dei passi indietro. Lo scorso anno in Inghilterra, in seguito a una sentenza giudiziaria, erano state infatti interrotte tutte le terapie in corso ed era stato istituito l'obbligo di sele- zione dei casi da trattare da parte di una Corte di Giustizia [9]. Tale decisione aveva di fatto esautorato il ruolo dell'équipe medica e negato la validità del consenso informato alla terapia di minore e famiglia rendendo molto più difficile l'accesso a tali trattamenti. Per concludere possiamo quindi dire che esiste ancora un rilevante gender gap culturale ma anche una carenza di attenzione e formazione nel mondo scientifico pediatrico che speriamo possano essere colmati nel prossimo futuro.

\section{Bibliografia}

1. American Psychiatric Association. Manuale diagnostico e statistico dei disturbi mentali (DSM-5). Milano, 20I4 (5).

2. Osservatorio Nazionale Identità di Genere (ONIG). Standard sui percorsi di affermazione di genere nell'ambito della presa in carico delle persone transgender e gender nonconforming (TGNC). http:// www.onig.it/node/I9

3. World Professional Association of Transender Health (WPATH). Standards of care version 7. https://www.wpath.org/publications/ soc

4. G. Tornese et al. Disforia di genere e dintorni. Medico e Bambino 20I6;35:437-444.

5. Lowry R, Johns MM, Gordon AR, et al. Nonconforming Gender Expression and Associated Mental Distress and Substance Use Among High School Students. JAMA Pediatr. 2018 Nov I;I72(II):IO2O-IO28.

6. Turban JL , King D, Carswell JM, Keuroghlian AS. Pubertal Suppression for Transgender Youth and Risk of Suicidal Ideation. Pediatrics. $2020 \mathrm{Feb} ; \mathrm{I} 45(2)$ :e20I9I725.

7. Determina AIFA 2I756/20I9. https:/www.gazzettaufficiale.it/eli/ $\mathrm{id} / 2019 / 03 / 02 /$ I9AOI426/SG

8. Determina AIFA I04272/2020 e I04273/2020. https://www. gazzettaufficiale.it/atto/serie_generale/caricaDettaglioAtto/originario?atto.dataPubblicazioneGazzetta $=2020-09$-30\&atto.codiceRedazionale $=20$ A05244\&elenco3ogiorni=false; https: $/ / \mathrm{www}$. gazzettaufficiale.it/atto/serie_generale/caricaDettaglioAtto/originario?atto.dataPubblicazioneGazzetta $=2020-09-30 \&$ atto.codiceRedazionale $=20$ Ao 5245 \&elenco 3 ogiorni $=$ true

9. Barbi L, Tornese G. Puberty blockers in gender dysphoria: an international perspective. Arch Dis Child. 202I Jul 27:archdischild-202I-32I960. doi: I0.II36/archdischild-202I-321960.

ccentenari@gmail.com

\section{La miopia del ventunesimo secolo}

\section{blister}

Abbiamo una fiducia quasi incondizionata sulla perfezione della natura ed è per questo che talvolta ci sorprendiamo di difetti del corpo umano incompatibili con una speranza di sopravvivenza. Per esempio ci meravigliamo di come il nostro occhio, organo estremamente raffinato, sembri in realtà difettoso in gran parte della popolazione infantile; negli ultimi quattro decenni la prevalenza di miopia nella popolazione pediatrica è progressivamente aumentata fino a raggiungere percentuali dell' $85 \%$ in alcune popolazioni asiatiche. Nella maggior parte della nostra esistenza come specie homo sapiens, passata da cacciatori-raccoglitori nelle savane e nelle foreste, la visione sfocata avrebbe significato un grave rischio per la sopravvivenza; e, in questa logica, gli occhiali sono una moderna pezza culturale per l'errore di progettazione della natura. II problema in verità potrebbe essere l'ambiente del ventunesimo secolo molto più ostile rispetto a quello del nostro passato quando per tetto avevamo le stelle e non c'era necessità di indossare lenti correttive. Una revisione sistematica ha raccolto 33 studi per valutare l'associazione tra la miopia e l'uso di tecnologie digitali (telefonino, tablet, computer) nella popolazione 3-33 anni. II tempo passato con i device da soli o associati all'uso del computer aumenta il rischio di miopia (rispettivamente OR 1,26, IC 95\% 1,00, 1,60 e OR 1,77, IC 95\% 1,28, 2,45) nella popolazione con età media di 3-16 anni [1]. Al di là dei problemi metodologici della revisione causati dall'ampia eterogeneità degli studi, questi dati confermano la preoccupazione sullo stato di salute della vista dei nostri bambini: un'epidemia di miopia che sta diventando un serio problema di sanità pubblica. Non si conoscono con precisione i fattori causali di questa epidemia di distorsione del globo oculare, come il momento di maggior sensibilità o criticità dell'occhio sottoposto a una continua sollecitazione di messa a fuoco da vicino, ma i dati sulle nuove abitudini di vita correlati all'esito sulla vista si accumulano. In Europa l'età più a rischio per la progressione della miopia è quella della scuola primaria, e la precoce esposizione del lattante alla luce solare previene questo disturbo visivo; inoltre il vivere all'aria aperta e l'illuminazione solare riducono il rischio di miopia nel bambino [2]. Per ogni ora della settimana che il bambino passa all'aperto si riduce il rischio di mettere gli occhiali: questa è una guida anticipatoria che ogni genitore dovrebbe conoscere.

1. Foreman J, Salim AT, Praveen A, et al. Association between digital smart device use and myopia: a systematic review and meta-analysis. Lancet Digit Health. 202I Dec;3(I2):e806-e8I8.

2. Tricard D, Marillet S, Ingrand P, et al. Progression of myopia in children and teenagers: a nationwide longitudinal study. Br J Ophthalmol. 202I Mar I2;bjophthalmol-2020-318256. 